\section{Commentary: Computational approaches for valve repair: Calculating the unknown}

\author{
Edgar Aranda-Michel, BS, ${ }^{\mathrm{a}}$ and \\ Ibrahim Sultan, MD ${ }^{\mathrm{a}, \mathrm{b}}$
}

Mitral valve regurgitation (MR) is the second most common valve pathology, preceded only by aortic stenosis. ${ }^{1}$ Standardization of surgical repair in this cohort is challenging, given the various etiologies and a variety of valve repair techniques. This is particularly prevalent with neochordae implantation. In this issue of the Journal, Caballero and colleagues ${ }^{2}$ use finite element analysis to examine the hemodynamic and biomechanical results of neochordae attachment profiles via a transapical approach. The most important finding in this paper is that while various attachment techniques resulted in resolution of $\mathrm{MR}$, there were drastic differences in the tension and stress in the chordae and leaflet, respectively. This emphasizes that while the clinical results may be equivalent, the underlying mechanical consequences can differ substantially, a metric that is only obtainable via such computational methods.

All models are wrong, but some are useful. ${ }^{3}$ This is an adage that is tied to any model development or analysis. It is paramount to interpret the results of any simulation in the context of the simplifications made in constructing the model. For this model, 2 main simplifications were used: (1) the aortic and mitral annulus were held constant throughout the cardiac cycle and (2) the left ventricular

From the a Division of Cardiac Surgery, Department of Cardiothoracic Surgery, University of Pittsburgh, Pittsburgh, Pa; and ${ }^{\mathrm{b}} \mathrm{Heart}$ and Vascular Institute, University of Pittsburgh Medical Center, Pittsburgh, Pa

Disclosures: The authors reported no conflicts of interest.

The Journal policy requires editors and reviewers to disclose conflicts of interest and to decline handling or reviewing manuscripts for which they may have a conflict of interest. The editors and reviewers of this article have no conflicts of interest.

Received for publication June 4, 2020; revisions received June 4, 2020; accepted for publication June 10, 2020; available ahead of print July 8, 2020.

Address for reprints: Ibrahim Sultan, MD, Division of Cardiac Surgery, Department of Cardiothoracic Surgery, University of Pittsburgh, Center for Thoracic Aortic Disease, Heart and Vascular Institute University of Pittsburgh Medical Center, 5200 Centre Ave, Suite 715, Pittsburgh, PA 15232 (E-mail: sultani@upmc.edu). JTCVS Open 2020;3:46-7

2666-2736

Copyright $@ 2020$ The Authors. Published by Elsevier Inc. on behalf of The American Association for Thoracic Surgery. This is an open access article under the CC BY-NCND license (http://creativecommons.org/licenses/by-nc-nd/4.0/).

https://doi.org/10.1016/j.xjon.2020.06.005

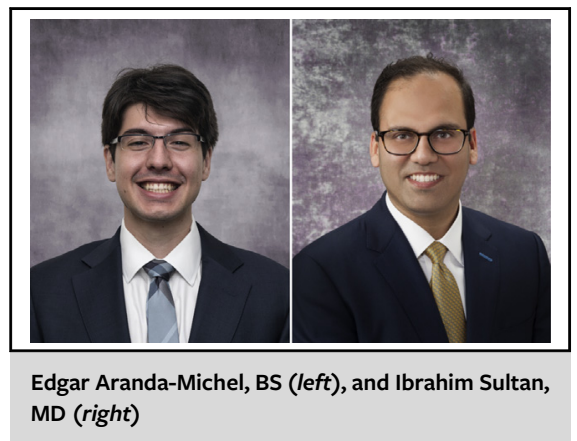

CENTRAL MESSAGE

While the clinical outcomes of different approaches to valve repair may be similar, the biomechanical results can be drastically different.

cavity was treated as a moving boundary condition, meaning that the endocardium of the left ventricle moves the same amount and at the same rate regardless of the applied tensions. It is important to note that these simplifications do not invalidate the findings or merit of the simulation, they only serve to appropriately interpret the results.

The authors found that when repairing a P2/P3 prolapse with an anterolateral approach, there was a substantial increase in the tension and stress. Although the magnitude of these increases is striking, it must be placed in the context of the simulation. It has been well established that the conformational change of the mitral annulus as well as the dynamism between the aortic and mitral annulus play a key role in mitral valve coaptation. ${ }^{4}$ As such, these increases in stress are likely an overestimate due to the model simplification. Nevertheless, this is unlikely to completely account for the increase in stress. This highlights a fundamental need of all simulation-based approaches, revalidation in in vivo models.

Neochordae implantation can be a challenging procedure, and one may need more than 100 procedures to overcome the initial learning curve. ${ }^{5}$ One critical component to appreciate is the tension that is applied to the neochordae when tied. A very small subjective difference in neochord length may not present with a significant recurrence of MR. This finding is echoed by the results of this paper, where optimal neochord length versus a 5\% reduction in length resulted in no MR. However, while the clinical results are comparable, these authors show that the biomechanics are quite disparate, with more than a $300 \%$ increase in systolic stress in the 
overtightened case. Again, this is likely an overestimation. However, a large increase in tension is not unexpected, given the pretension that is applied and the hyperelastic nature of the neochordae. This large increase in tension could have negative remodeling effects over the long term. Although this tension is difficult to measure, groups have been working on devices to assess the tension intraoperatively. ${ }^{6}$ Intraoperative measurements can be applied to the simulation to calculate stress and with long-term patient follow-up, the calculated stress can be correlated with remodeling of the mitral annulus and the LV cavity.

Simulations are needed to capture the wide range of pathologies in primary valvular regurgitation. Differing geometries can dramatically change the biomechanics throughout the cardiac cycle. The principle behind this paper is that increases in stress should ideally be avoided when performing a neochordae repair for primary mitral regurgitation. Establishing a cut-off value for stress is important so that a full design space can be explored in this setting. This manuscript represents a first step in performing complex simulations to evaluate neochordae implantation with the hopes of using it to drive operative decision-making.

\section{References}

1. Wu S, Chai A, Arimie S, Mehra A, Clavijo L, Matthews RV, et al. Incidence and treatment of severe primary mitral regurgitation in contemporary clinical practice. Cardiovasc Revasc Med. 2018;19:960-3.

2. Caballero A, McKay R, Sun W. Computer simulation of transapical mitral valve repair with neochordae implantation: clinical implications. J Thorac Cardiovasc Surg Open. 2020;3:27-44.

3. Box GEP. Science and statistics. J Am Stat Assoc. 1976;71:791-9.

4. Lawrie GM. Structure, function, and dynamics of the mitral annulus: importance in mitral valve repair for myxamatous mitral valve disease. Methodist Debakey Cardiovasc J. 2010;6:8-14.

5. Holzhey DM, Seeburger J, Misfeld M, Borger MA, Mohr FW. Learning minimally invasive mitral valve surgery: a cumulative sum sequential probability analysis of 3895 operations from a single high-volume center. Circulation. 2013;128:483-91.

6. Grinberg D, Le M-Q, Kwon YJ, Fernandez MA, Audigier D, Ganet F, et al Mitral valve repair based on intraoperative objective measurement. Sci Rep. 2019;9:4677. 